Article

\title{
Effect of Geraniol and Citronellol Essential Oils on the Biophysical Gating Properties of AMPA Receptors
}

\author{
Mohammad Qneibi ${ }^{1, *(\mathbb{D})}$, Nidal Jaradat ${ }^{2}\left(\mathbb{D}\right.$ and Nour Emwas ${ }^{1}$ \\ 1 Department of Biomedical Sciences, Faculty of Medicine and Health Sciences, An-Najah National University, \\ Nablus 00970, Palestine; noourryy@gmail.com \\ 2 Department of Pharmacy, Faculty of Medicine and Health Sciences, An-Najah National University, \\ Nablus 00970, Palestine; nidaljaradat@najah.edu \\ * Correspondence: mqneibi@najah.edu; Tel.: +972-545-975-016
}

Received: 28 August 2019; Accepted: 25 October 2019; Published: 4 November 2019

\begin{abstract}
Essential oils have been advertised endlessly to be very beneficial for the health of humans, and an extensive amount of research examines the validity of such claims. In contribution, the current study evaluates the neuroprotective properties of Citronellol and Geraniol essential oils (EOs). In relationship to the biophysical gating properties of different the $\alpha$-amino-3-hydroxy-5-methyl-4-isoxazolepropionic acid receptor (AMPAR) subunits, the EOs were administered to HEK293 (Human embryonic kidney 293) cells and examined for any inhibition and effect on desensitization or deactivation rates, using whole-cell patch-clamp electrophysiology. Our results demonstrated the highest levels of inhibition from Citronellol oil by four-fold on all AMPARs subunits. Likewise, Geraniol oil had a similar inhibiting impact on the receptors, and both oils decreased the desensitization and deactivation rates of the inhibited receptors. Thus, the examined EOs of this study portray neuroprotective qualities by targeting AMPARs activation and reducing desensitization and deactivation rates. Finally, the results of the current study entail a better understanding of AMPARs, provides a natural template for future drug synthesis to treat neurological diseases associated with excessive AMPAR activation, and offers a possible mechanism by which these essential oils deploy their 'calming' effect.
\end{abstract}

Keywords: biophysical gating properties; essential oils; inhibition; neuroprotective; AMPA receptors

\section{Introduction}

Essential oils (EOs) are usually extracted from plants and are used for aromatherapy, which is an alternative medicine that demonstrates the healing effect to the aromatic compounds presented in the oil [1,2]. Although its therapeutic potential remains controversial, the usage of essential oil has been increasingly popular in the general population [3]. Many EOs are currently being presented to showcase a wide range of benefits; two important EOs are the Citronellol and Geraniol oils. Citronellol oil is obtained from different species of lemongrass (Cymbopogon) and is mostly composed of Citronellol and Geraniol, which are found in over 70 other plants [4]. To the contrary, Geraniol oil is composed of different extracted molecules from the essential oils of various plants, including rose, geranium, and palmarosa species [4-7].

The health benefits of Citronellol include antibacterial, antidepressant, antiseptic, antispasmodic, anti-inflammatory, deodorant, diaphoretic, diuretic, febrifuge, fungicidal, insect repellent, stomachic, stimulant, tonic, and vermifuge effects. Although Geraniol is composed of a combination of plants, its health benefits are of similar to those observed by Citronellol oil [5-7]. Accumulating evidence suggests that both of these oils have a calming effect and reduce anxiety, stress, and depression, yet their neuroprotective properties remain obscure $[3,6]$. Hence, the current study evaluates these EOs for 
neuroprotective qualities to the activity of the $\alpha$-amino-3-hydroxy-5-methyl-4-isoxazolepropionic acid receptor, AMPARs.

AMPARs are part of the ionotropic glutamate receptor family (iGluRs) that respond to glutamate. These receptors are responsible for the majority of the fastest excitatory neurotransmission and play a significant role in important brain activity, such as learning and memory formation [8,9]. However, excessive AMPARs have been associated with neurotoxicity, which is linked to various neurodegenerative diseases. An increasing body of evidence suggests the progression and pathogenesis of several diseases are related to the mechanism of neuronal death caused by excitotoxicity [10]. Thus, glutamate-induced excitotoxicity has been implicated in many different neurological disorders, such as epilepsy, ischemia, amyotrophic lateral sclerosis (ALS), and Alzheimer's disease (AD) [11,12].

In an attempt to treat various chronic neurodegenerative disorders, multiple studies researched the possibilities of potential AMPAR antagonists to reduce the excitotoxicity [8,13]. In fact, due to drug resistance, a new target for anti-epileptic and anti-seizures is towards AMPAR inhibition, precisely drugs that act as non-competitive inhibitors [12-16]. However, many of the researched antagonists fail at the clinical trials due to low efficacy or severe side effects, such as respiratory depression, sedation, tolerance, dependence, and extrapyramidal symptoms [14]. Moreover, many of the possible drug candidates for AMPARs possess selectivity towards specific AMPAR subunits, which fails to be effective in many diseases, such as ALS [13]. Research is currently fixated on the neuroprotective properties of various essential oils and natural components to counteract undesired side effects [17-20]. The current study investigates the possible mechanism for such an effect in AMPAR inhibition. Moreover, through a natural approach, we aim to investigate the potential possibility of these EOs on inhibiting the activity of AMPARs and provide a possible drug candidate for diseases associated with excessive AMPAR activation. Equally as important, the results of this study shed light on the protein-receptor interaction and allow a better understanding of AMPARs. The inhibition of AMPARs was calculated by using an electrophysiological approach to record the whole-cell current (patch clamp), with and without the administration of EOs into human embryonic kidney cells HEK293. The same methodology was also applied to investigate the effect of these EOs on other biophysical gating properties, i.e., the desensitization and deactivation rates of AMPARs. Alteration in the kinetics of the receptor's desensitization and deactivation rates can also signify the potential of the oils in drug synthesis. Finally, the study will examine these EOs individually on the most abundant homomeric and heterologous AMPAR subunits; GluA1, GluA2, GluA1/2, and GluA2/3.

\section{Material and Methods}

\subsection{HEK293 Cell Patch-Clamp Recordings}

The whole-cell patch-clamp technique was used in the electrophysiology recordings of HEK293 cells to obtain the activation, desensitization, and deactivation rates of the expressed AMPARs. Using Sutter's instrument; the integrated patch amplifier (IPA, Novato, CA 94949, USA), the whole-cell recordings were optimized, and data acquisition was analyzed using Igor Pro7 (Wave Metrics, Inc. Lake Oswego, OR 97036, USA). SutterPatch Software v. 1.1.1 (Sutter Instruments, Novato, CA 94949, USA) was used to replicate the cells' membrane currents; hence, the sampling frequency was set at $10 \mathrm{kHz}$, while the low-pass filter was set to $2 \mathrm{kHz}$. Borosilicate glass was used to fabricate the patch electrodes with a resistance of 2-4 M . The extracellular solution contained (values are in $\mathrm{mM}$ ): $150 \mathrm{NaCl}, 2.8 \mathrm{KCl}, 0.5 \mathrm{MgCl}_{2}, 2 \mathrm{CaCl}_{2}, 10 \mathrm{HEPES}$ adjusted to $\mathrm{pH} 7.4$ with $\mathrm{NaOH}$. Upon succeeding a gigaseal with an AMPAR expressed HEK293 cell, it was interchangeably exposed to three different solutions; the first contained the glutamate alone, the second contained glutamate + Citronellol while the last tube contained glutamate + Geraniol. The cell was supplied with these solutions one at a time, exchanged every $500 \mathrm{~ms}$, from a double barrel glass (theta tube) that was also constantly supplying the cell with a solution of $110 \mathrm{CsF}, 30 \mathrm{CsCl}, 4 \mathrm{NaCl}, 0.5 \mathrm{CaCl}_{2}, 10$ Trypsin EDTA solution B (0.25\%), EDTA $(0.05 \%), 10$ HEPES, adjusted to $\mathrm{pH} 7.2$ with $\mathrm{CsOH}$ (values are in $\mathrm{mM}$ ), from the other barrel. The theta 
tube was mounted on a high-speed piezo solution switcher (AutoMate Scientific, Inc. Berkeley, CA 94710, USA) to mimic the speed of naturally-induced AMPAR currents. The control were the baseline currents produced from supplying the cell with glutamate alone, tested and recorded before and after each exposure of the cell to the oils. AMPAR-current deactivation and desensitization were fitted with two exponentials, and the weighted tau $(\tau \mathrm{w})$ was calculated as $\tau \mathrm{w}=(\tau \mathrm{f} \times \mathrm{af})+(\tau \mathrm{s} \times$ as $)$, where (af) and (as) are the relative amplitudes of the fast ( $\tau \mathrm{f})$ and slow $(\tau \mathrm{s})$ exponential component. Hence, the currents were evoked by the application of $3 \mathrm{mM}$ glutamate for desensitization while for deactivation, the cells were administered $1 \mathrm{mM}$ of glutamate for $500 \mathrm{~ms}$. To compare the current before and after the administration of the EOs, the currents (I) at the steady-state was normalized to the current obtained with agonist alone $\left(\mathrm{I}_{0}\right)$. The same concept follows for the currents obtained from separately and individually treating the cell with the selected EOs. Inhibition was calculated as a percentage of the difference in current amplitude from the pulse before to antagonist application and the second pulse after the current stabilization post-antagonist application. Significance compared with AMPAR expressed alone or with AMPAR+ compound of interest; $p$-value (one-way ANOVA): $*<0.05,{ }^{* *}<0.01,{ }^{* * *}<0.001$, ns: not significant.

\section{2. cDNA Transient Transfection in HEK293 Cells}

All AMPARs subunits used in this experiment are in the flip isoform (pBlueScript), which were obtained from S. F. Heinemann (Salk Institute, La Jolla, CA, USA) and subcloned in pRK for expression in HEK293. P. H. Seeburg (Max Planck Institute for Medical Research, Heidelberg, Germany) gifted the laboratory with the GluA2 unedited form (R607Q) (flip isoforms) and enhanced green fluorescent protein (EGFP) in pRK5. Homomeric AMPAR plasmids were cotransfected with a GFP expression vector $(1 \mu \mathrm{g}$ of GluA1 or GluA2, $1 \mu \mathrm{g}$ GFP) in HEK293 cells by chemical-mediated transfection. Likewise, heterologous AMPAR plasmids were transfected in the same manner under a 1:1.2 ratio. Each cell had a specific AMPAR subunit expressed, to maintain the level of expression throughout the experiment and to individually examine the effect of the oils on the specific receptor, be it homomeric or heteromeric. Cells were then seeded in Petri dishes in DMEM supplemented with $10 \%$ foetal calf serum and antibiotics and maintained in a humidified incubator at $37^{\circ} \mathrm{C}$ and $5 \% \mathrm{CO}_{2}$. Highly fluorescent cells were identified and selected for recording. Cells of similar initial current, produced from the glutamate alone before oil exposure, were used in the sample size to reduce the range of variability in AMPAR expression and as a result, current discrepancies.

\subsection{HEK293 Cell Culture and Transfection}

The electrophysiology recording occurred two to three days after transfecting the cells of a temperature and membrane potential set at $22{ }^{\circ} \mathrm{C}$ and $-60 \mathrm{mV}$, respectively. For the purpose of cell culturing and transfection all HEK293 cells (Sigma, 89555 Steinheim am Albuch, Germany) were grown in a medium of DMEM, Dulbecco Modified Eagle Medium, (Sigma, Ronkonkoma, NY 11779, USA) which contained $0.1 \mathrm{mg} / \mathrm{mL}$ streptomycin, and $1 \mathrm{mM}$ sodium pyruvate (Biological Industries, Beit-Haemek, Israel) and a 10\% of FBS, foetal bovine serum. Before transfection, the cells were sub-cultured twice a week and left in the incubator for 24 hours, set at $37^{\circ} \mathrm{C}, 5 \% \mathrm{CO}_{2}$. After DNA preparation and cell culturing the transient cDNA was transfected in HEK293 cells. The transfection reagent that was used was jetPRIME (Polyplus: New York, NY 11779, USA) after which the cells were left for $36 \mathrm{hrs}$ in the incubator. Finally, to prepare the cells for whole-cell patch-clamp, they were replanted from the 12-well plates to coverslips coated with Laminin $(1 \mathrm{mg} / \mathrm{mL}$; Sigma, Steinheim am Albuch, Germany). 


\section{Results and Discussion}

\subsection{Effect of Oils on the Peak Current of AMPARs}

To inquire direct effects of the essential oils on amplitude generated by AMPARs, Heterologous expression (GluA1/2 and GluA2/3) and Homologous expression of GluA1 and GluA2 subunits in HEK293 cells were individually and separately examined via whole-cell patch-clamp with and without the administration of the compound of interest. The peak currents of AMPA receptors subunits with glutamate alone and no treatment are $834 \pm 45$ pA, $1330 \pm 77$ pA, $520 \pm 39$ pA, $443 \pm 45$ pA, for GluA1, GluA2, GluA1/2, and GluA2/3, respectively as shown in Figure 1A. The essential Citronellol oil was applied to the cell and resulted in a drastic reduction in the peak current of the AMPARs previously mentioned to $185 \pm 23$ pA, $380 \pm 26$ pA, $121 \pm 19$ pA, and $153 \pm 32$ pA. Likewise, the effect of Geraniol oil had a similar impact on the peak current of AMPAR subunits. Hence, the peak current of GluA1, GluA2, GluA1/2, and GluA2/3 were reduced to the following readings; $232 \pm 31 \mathrm{pA}, 429 \pm 35 \mathrm{pA}$, $163 \pm 17$ pA, and $164 \pm 35$ pA Figure 1B showcases whole-cell current traces from GluA1 channels (most significant) expressed in HEK-293 cells in the absence (left) and presence (right) of Citronellol oil.

A

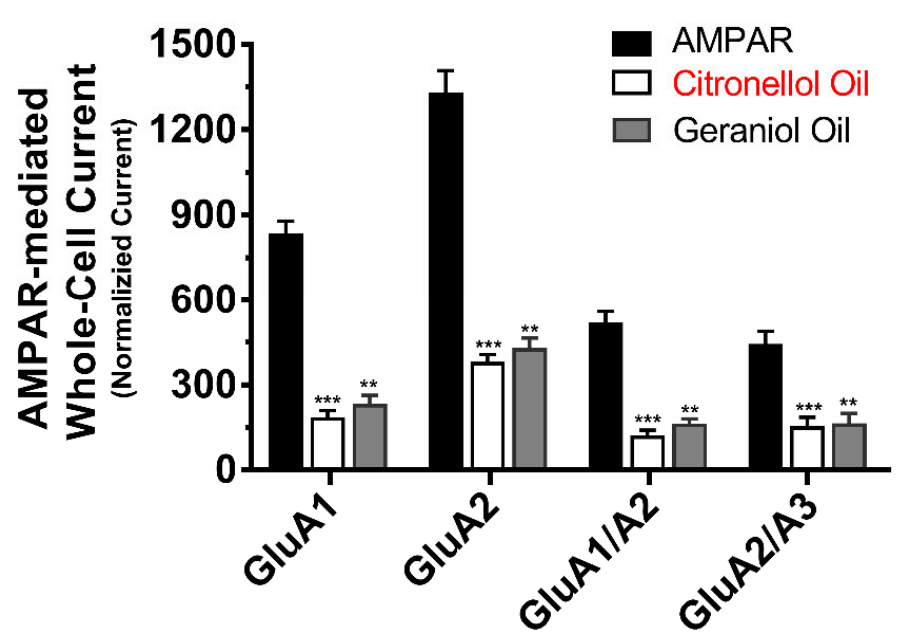

B

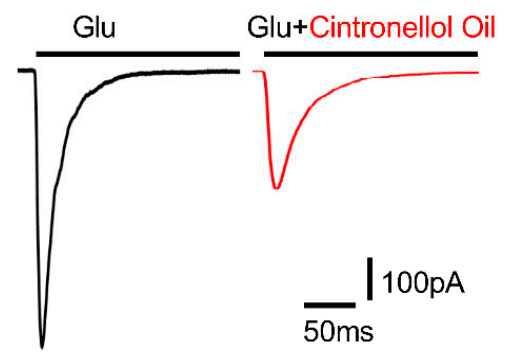

Figure 1. Effect of essential oils and compounds on the amplitude of the whole-cell generated by various AMPAR subunits. (A). Amplitude generated from glutamate alone (black), glutamate + Citronellol (white) and from glutamate + Geraniol (grey). (B). Pair of representative whole-cell current traces from GluA1 channels (most significant) expressed in HEK-293 cells in the absence (left) and presence (right) of Citronellol oil. The recording was conducted upon $500 \mathrm{~ms}$ application of $10 \mathrm{mM}$ glutamate to whole-cell recording from HEK293 cells expressing various AMPARs subunits.

The whole-cell current recording was conducted at $-60 \mathrm{mV}, \mathrm{pH} 7.4$, and $22^{\circ} \mathrm{C}$. Graphs summarize weighted time constants for activation. Data shown are mean $\pm S E M ; n=4$ (number of patch cells in the whole-cell configuration). A one-way ANOVA test was used to measure the significance: ${ }^{*} p<0.05$; ${ }^{* *} p<0.01 ;{ }^{* *} p<0.001 ;$ ns, not significant.

\subsection{Influence of Oils on the Desensitization Rate}

The Inhibition of the EOs on AMPAR activity shed light on the possibility of their impact on other biophysical gating properties of AMPARs such as desensitization (Figure 2). The average desensitization time of GluA1, GluA2, GluA1/2, and GluA2/3 were as follows; $2.9 \pm 0.1 \mathrm{~ms}, 2.4 \pm 0.1 \mathrm{~ms}$, $5.1 \pm 0.3 \mathrm{~ms}$, and $2.4 \pm 0.2 \mathrm{~ms}$ denoting an average rate $(\tau=1 / \mathrm{ms})$ of $0.34 \mathrm{~ms}^{-1}, 0.42 \mathrm{~ms}^{-1}, 0.20 \mathrm{~ms}^{-1}$, and $0.42 \mathrm{~ms}^{-1}$, respectively Figure 2A. A decrease in desensitization rate was in GluA1, GluA2 homologous AMPARs and GluA1/2 and GluA2/3 Heterologous AMPARs upon treating the cells with 
either EOs. Hence for Citronellol oil desensitization increased to $4.3 \pm 0.4 \mathrm{~ms}, 2.8 \pm 0.2 \mathrm{~ms}, 5.7 \pm 0.3 \mathrm{~ms}$, and $2.7 \pm 0.3 \mathrm{~ms}$, which read a decrease in the desensitization rates to $0.23 \mathrm{~ms}^{-1}, 0.36 \mathrm{~ms}^{-1}, 0.18 \mathrm{~ms}^{-1}$, and $0.37 \mathrm{~ms}^{-1}$, respectively Figure 2A. Likewise, for Geraniol oil, the desensitization reduced to $3.6 \pm 0.3 \mathrm{~ms}, 2.6 \pm 0.2 \mathrm{~ms}, 5.4 \pm 0.3 \mathrm{~ms}$, and $2.6 \pm 0.4 \mathrm{~ms}$ denoting a decrease in the rate of desensitization to equal; $0.28 \mathrm{~ms}^{-1}, 0.38 \mathrm{~ms}^{-1}, 0.18 \mathrm{~ms}^{-1}$, and $0.38 \mathrm{~ms}^{-1}$, respectively. Figure $2 \mathrm{~B}$ showcases the whole-cell current traces from GluA1 channels (most significant) expressed in HEK293 cells in the absence (left) and presence (right) of Citronellol oil.

A

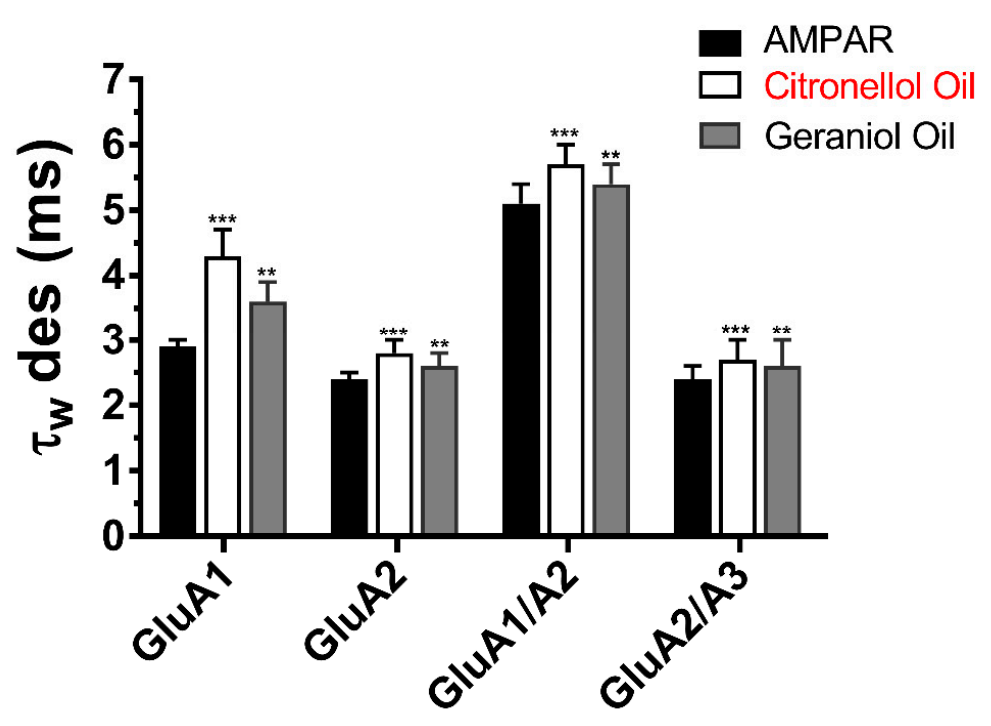

B

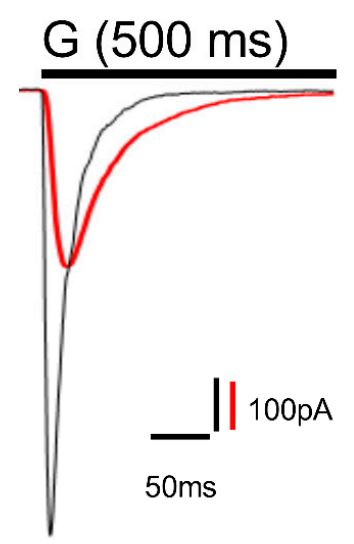

Figure 2. Effect of oils and compounds on AMPAR desensitization. (A). Desensitization from glutamate alone (black), glutamate + Citronellol (white) and from glutamate + Geraniol (grey). (B). Pair of representative whole-cell current traces from GluA1 channels (most significant) expressed in HEK-293 cells in the absence (left) and presence (right) of Citronellol oil. The recording was conducted upon $500 \mathrm{~ms}$ application of $10 \mathrm{mM}$ glutamate to whole-cell recording from HEK293 cells expressing various AMPARs subunits. Data shown are mean \pm SEM; $n=4$ (number of patch cells in the whole-cell configuration). A one-way ANOVA test was used to measure the significance: ${ }^{*} p<0.05 ;{ }^{* *} p<0.01$; *** $p<0.001 ;$ ns, not significant.

\subsection{Reduction in Deactivation Rate from Oils}

The effects of EOs on AMPAR's desensitization kinetics motivated the investigation of their influence on AMPAR deactivation (Figure 3). The average deactivation time for GluA1, GluA2, GluA1/2 and GluA2/3 recorded at; $2.3 \pm 0.1 \mathrm{~ms}, 2.1 \pm 0.1 \mathrm{~ms}, 2.5 \pm 0.2 \mathrm{~ms}, 2.5 \pm 0.3 \mathrm{~ms}$, denoting an average rate of $0.43 \mathrm{~ms}^{-1}, 0.48 \mathrm{~ms}^{-1}, 0.40 \mathrm{~ms}^{-1}, 0.40 \mathrm{~ms}^{-1}$, respectively. Upon treating the cells with Citronellol oil, the deactivation rates decreased for all AMPARs subunits. Hence, the deactivation was recorded at $3.7 \pm 0.2 \mathrm{~ms}\left(0.27 \mathrm{~ms}^{-1}\right), 3.2 \pm 0.3 \mathrm{~ms}\left(0.31 \mathrm{~ms}^{-1}\right), 3.4 \pm 0.2 \mathrm{~ms}\left(0.29 \mathrm{~ms}^{-1}\right)$ and $2.9 \pm 0.4 \mathrm{~ms}$ $\left(0.34 \mathrm{~ms}^{-1}\right)$ for GluA1, GluA2, GluA1/2 and GluA2/3, respectively. Likewise, upon treating the cells with Geraniol oil, the deactivation for GluA1, GluA2, GluA1/2, and GluA2/3 increased to the following; $3.2 \pm 0.4 \mathrm{~ms}\left(0.31 \mathrm{~ms}^{-1}\right), 2.8 \pm 0.1 \mathrm{~ms}\left(0.36 \mathrm{~ms}^{-1}\right), 3.1 \pm 0.5 \mathrm{~ms}\left(0.32 \mathrm{~ms}^{-1}\right)$, and $2.7 \pm 0.5 \mathrm{~ms}\left(0.37 \mathrm{~ms}^{-1}\right)$. 


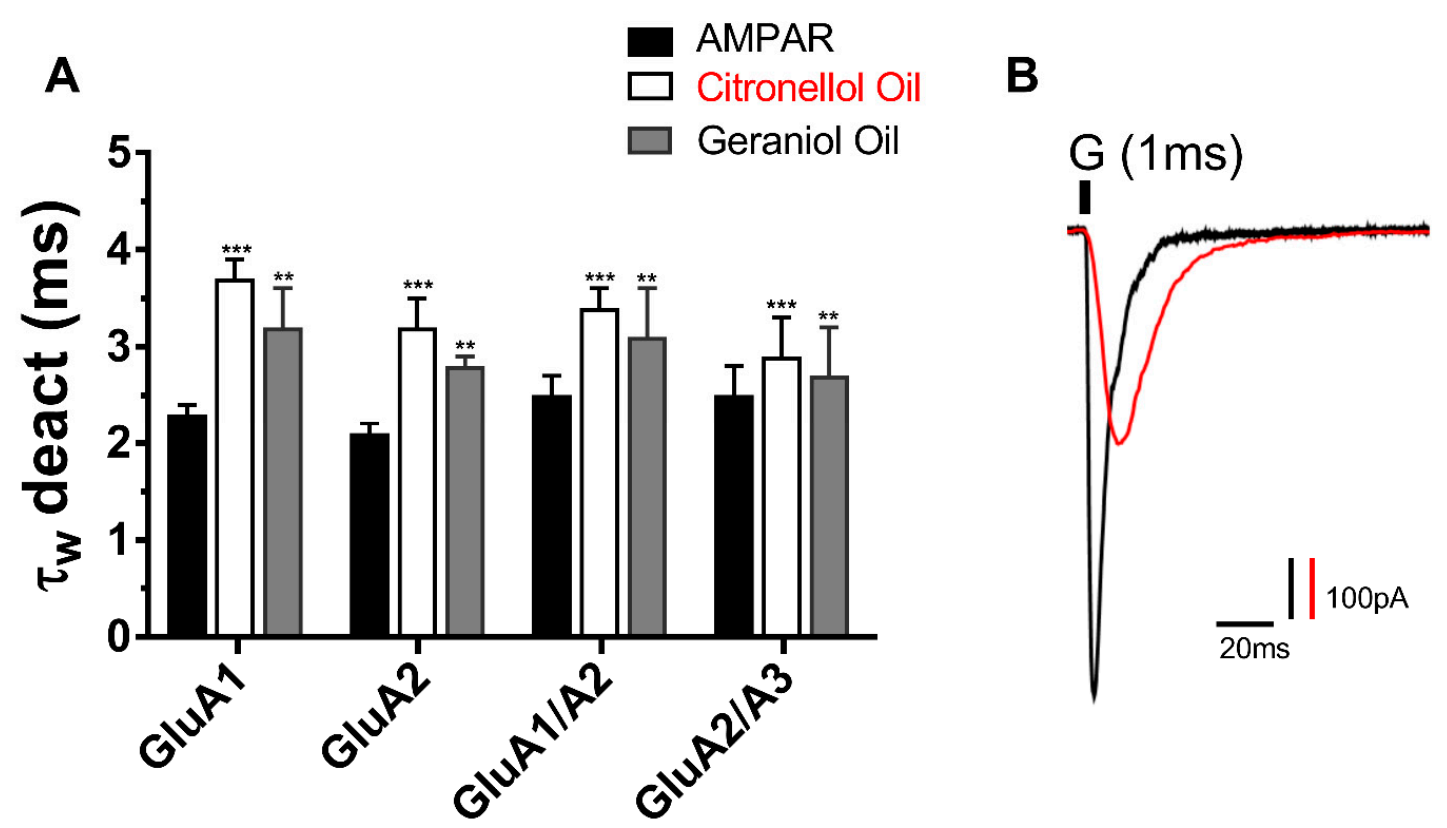

Figure 3. Effect of oils and compounds on AMPAR Deactivation. (A). The graphical representation that summarizes the weighted time constants for deactivation ( $\tau_{W}$ deact) for the whole cell recordings of all AMPARs subunits, conducted at $-60 \mathrm{mV}, \mathrm{pH} 7.4$, and $22{ }^{\circ} \mathrm{C}$, upon $1 \mathrm{~ms}$ application of $10 \mathrm{mM}$ glutamate alone (black), glutamate + Citronellol (white), and from glutamate + Geraniol (grey). (B). Pairs of representative whole-cell current traces from GluA1 channels (most significant) expressed in HEK-293 cells in the absence (left) and presence (right) of Citronellol oil. Data shown are mean \pm SEM; $\mathrm{n}=4$ (number of patch cells in the whole-cell configuration). A one-way ANOVA test was used to measure the significance: ${ }^{*} p<0.05 ;{ }^{* *} p<0.01$; ${ }^{* * *} p<0.001$; ns, not significant.

\section{Discussion}

The excessive activation of AMPARs has been associated with numerous neurological diseases; as a result, has been the target of many drugs. However, only one pharmaceutical drug is currently on the market, and is known as perampanel. This drug is primarily used as an antiepileptic drug, yet has numerous serious side effects, such as depression, anxiety, paranoia, weight gain, and joint and back pain [21]. In response, the current study investigates two conventional essential oils; the Citronellol and Geraniol oils as possible AMPAR antagonist. In an attempt to reduce the side effect seen from perampanel, these oils have been documented to be antidepressants, anti-inflammatory, diuretics, etc. Hence the result of the study indicates that not only do they inhibit AMPARs but provide a natural antagonist of no side effects. In the same manner as the reduction in peak current, the desensitization and deactivation rates also decreased, insinuating the action of EOs target all biophysical properties of the AMPAR subunits in the same way.

The level of inhibition between the EOS was distinct as the Citronellol oil inhibited AMPARs subunit by four folds while the Geraniol oil inhibition was by only three-fold. Moreover, the inhibition between AMPAR subunits was also biased in that specific subunits were more susceptible to inhibition than others hence, the AMPAR subunit that was significantly impacted the most was GluA1 followed by its heteromeric subunit GluA1/A2, then GluA2 and finally GluA2/3. Both oils portrayed the same bias to specific AMPAR subunits, suggesting the action of these oils is similar and target the same antagonist site. However, due to the discrepancy seen in inhibition, the Citronellol is a more favorable and stable antagonist for AMPARs than the Geraniol oil. Although both oils favor specific AMPAR subunits, they remained to significantly inhibit all tested subunits, which is critical significance because unlike Talampanel [22] that failed in clinical trials, due to its effectiveness on specific AMPAR subunits, these oils deploy their neuroprotective properties on all AMPARs. Finally, the inhibition of both 
essential oils was irrelevant to glutamate concentration, a suggestion they act as noncompetitive inhibitors. Due to excessive concentration of glutamate and as a result over activation of AMPARs, noncompetitive inhibitors are a significantly essential and desirable target in drug synthesis to treat the disease associated with AMPARs.

The administration of multi-components or single essential oils or their constituents in conventional medicine, complementary alternative medicine, folk medicine, and aromatherapy has been known for a long time, and its relevance is steadily growing [16-18]. In the last few years, many scientific investigations were established to assess the effectiveness and the mechanisms of action of these compounds on the central nervous system $[19,23,24]$. The results of the current study demonstrate the tested EOs implement their calming effects by directly inhibiting AMPARs but also reducing the desensitization and deactivation rates. The interaction between the oils and receptor is at a noncompetitive antagonist site that is crucial in the mechanism of the other AMPAR biophysical properties. Hence, desensitization is caused by ion channel pore closure without the disassociation of the ligand from the receptor. On the contrary, the deactivation of AMPARs is characterized as the natural decay in current after the dissociation of glutamate from the receptor and closure of the channel gate $[25,26]$. Computational ligand docking is needed to assess how the action of these oils targets all kinetics of the receptor's activation, deactivation, and desensitization. Nonetheless, reducing the desensitization and deactivation rates of AMPARs indirectly portrays neuroprotective properties as this will reduce the signal transduction and excessive activation of AMPARs, which is of pharmaceutical significance in treating epilepsy, ALS, and other neurological diseases.

However, only one pharmaceutical drug is currently in the market and is known as Perampanel. This drug is primarily used as an antiepileptic drug, yet has numerous serious side effects such as depression, anxiety, paranoia, weight gain, and joint and back pain [21]. In response, the current study investigates two conventional essential oils; the Citronellol and Geraniol oils as possible AMPAR antagonist. In attempt to reduce the side effect seen from Perampanel these oils have been documented to be antidepressant, anti-inflammatory, diuretics etc., hence the result of the study indicates that not only do they inhibit AMPARs, but also provide a natural antagonist of no side effects. In the same manner as the reduction in peak current, the desensitization and deactivation rates also decreased, insinuating the action of EOs target all biophysical properties of the AMPAR subunits in the same way.

The level of inhibition between the EOS was distinct as the Citronellol oil inhibited AMPARs subunit by four folds while the Geraniol oil inhibition was by only three-fold. Moreover, the inhibition between AMPAR subunits was also biased in that specific subunits were more susceptible to inhibition than others hence, the AMPAR subunit that was significantly impacted the most was GluA1 followed by its heteromeric subunit GluA1/A2, then GluA2 and, finally, GluA2/3. Both oils portrayed the same bias to specific AMPAR subunits, suggesting the action of these oils is similar and target the same antagonist site. However, due to the discrepancy seen in inhibition, the Citronellol is a more favorable and stable antagonist for AMPARs than the Geraniol oil. Although both oils favor certain AMPAR subunits, they remained to significantly inhibit all tested subunits, which is critical significance because unlike Talampanel that failed in clinical trials, due to its effectiveness on certain AMPAR subunits [22], these oils deploy their neuroprotective properties on all AMPARs. Finally, the inhibition of both essential oils was irrelevant to glutamate concentration, suggestion they act as non-competitive inhibitors. Due to excessive concentration of glutamate and as a result over activation of AMPARs, noncompetitive inhibitors are a significantly important and desirable target in drug synthesis to treat the disease associated with AMAPRs.

The administration of multi-components or single essential oils or their constituents in conventional medicine, complementary/alternative medicine, folk medicine, and aromatherapy has been known for a long time, and its relevance is steadily growing $[17,18]$. In the last few years, many scientific investigations have been established to assess the effectiveness and the mechanisms of action of these compounds on the central nervous system $[19,20]$. Previously, we have shown no inhibitory effect from Origanum syriacum L. and Lavandula dentate L. essential oils on any of the tested AMPAR subunits [6]. 
Although they influenced the desensitization and deactivation of the AMPARs, the results given previously were not as significant in comparison to the present oils that non-competitively inhibit AMPARs. The results of the current study demonstrate the tested EOs implement their calming effects by directly inhibiting AMPARs but also reducing the desensitization and deactivation rates. The interaction between the oils and receptor is at a noncompetitive antagonist site that is crucial in the mechanism of the other AMPAR biophysical properties. Hence, desensitization is caused by ion channel pore closure without the disassociation of the ligand from the receptor. On the other hand, the deactivation of AMPARs is characterized as the natural decay in current after the dissociation of glutamate from the receptor and closure of the channel gate $[14,16]$. Computational ligand docking is needed to assess how the action of these oils targets all kinetics of the receptor's activation, deactivation and desensitization. Nonetheless, reducing the desensitization, and deactivation rates of AMPARs may indirectly portray neuroprotective properties, as this will reduce the signal transduction and excessive activation of AMPARs, which is of pharmaceutical significance in treating epilepsy, ALS, and other neurological diseases.

\section{Conclusions}

In conclusion, our study provides an alternative natural template for drug synthesis that targets AMPARs of all subunits. Additionally, we provide a mechanism by which these oils deploy their calming effect and reduced anxiety. Previously reported implications in excessive activation or overexpression of AMPARs in various neurological diseases encouraged research to target AMPARs inhibition, which, as a result, act as anticonvulsant treatment. Only one FDA approved an anticonvulsant drug, proposed to have neuroprotective properties by inhibiting and targeting AMPARs, is currently on the market with numerous side effects. Hence, this paper provides evidence of the tested EO's possessing similar neuroprotective properties yet are non-synthetic. Moreover, we notice an inhibition of all tested subunits but biased favoritism for the calcium-permeable AMPAR subunits than calcium impermeable. This is significant in understanding the neurological disorders related to a dysregulation in AMPAR subunit expression and a better aim to target the specific subunits as well as understand the difference in structure-protein interaction between various AMPARs. Finally, the study also enhanced the potential neuroprotective properties of the tested EOs as they also decreased the desensitization and deactivation rates of AMPARs. We hope to continue this study in induced ALS rodents and report the effect of the EOs on the progression of the disease.

Author Contributions: Conceptualization, M.Q. and N.J.; funding acquisition, M.Q.; investigation, M.Q., N.J. and N.E.; methodology, M.Q. and N.J.; writing—original draft, M.Q.; writing—review and editing: M.Q., N.J. and N.E.

Funding: This work was supported by a grant from An-Najah National University (grant number: ANNU-1920-Sc011).

Acknowledgments: The authors would like to acknowledge An-Najah National University and the Dean of Scientific Research for their support and funding.

Conflicts of Interest: There are no conflicts of interest.

\section{References}

1. Almeida, L.d.F.D.d.; Paula, J.F.d.; Almeida, R.V.d.; Williams, D.W.; Hebling, J.; Cavalcanti, Y.W. Efficacy of citronella and cinnamon essential oils on Candida albicans biofilms. Acta Odontol. Scand. 2016, 74, $393-398$. [CrossRef] [PubMed]

2. Gandhi, G.R.; Vasconcelos, A.B.S.; Haran, G.H.; Calisto, V.K.D.S.; Jothi, G.; Quintans, J.S.S.; Cuevas, L.E.; Narain, N.; Júnior, L.J.Q.; Cipolotti, R.; et al. Essential oils and its bioactive compounds modulating cytokines: A systematic review on anti-asthmatic and immunomodulatory properties. Phytomedicine 2019, 31, 152854. [CrossRef] [PubMed]

3. Heghes, S.C.; Vostinaru, O.; Rus, L.M.; Mogosan, C.; Iuga, C.A.; Filip, L. Antispasmodic effect of essential oils and their constituents: A review. Molecules 2019, 24, 1675. [CrossRef] [PubMed] 
4. Lowring, L.M. Using therapeutic essential oils to support the management of anxiety. J. Am. Assoc. Nurse Pract. 2019, 31, 558-561. [CrossRef] [PubMed]

5. Qneibi, M.S.; Micale, N.; Grasso, S.; Niu, L. Mechanism of inhibition of GluA2 AMPA receptor channel opening by 2, 3-benzodiazepine derivatives: Functional consequences of replacing a 7, 8-methylenedioxy with a 7, 8-ethylenedioxy moiety. Biochemistry 2012, 51, 1787-1795. [CrossRef]

6. Qneibi, M.; Jaradat, N.; Hawash, M.; Naser Zaid, A.; Natsheh, A.-R.; Yousef, R.; Abuhasan, Q. The neuroprotective role of Origanum syriacum L. and Lavandula dentata L. essential oils through their effects on AMPA receptors. BioMed Res. Int. 2019, 2019, 5640173. [CrossRef]

7. Qneibi, M.; Hamed, O.; Fares, O.; Jaradat, N.; Natsheh, A.R.; AbuHasan, Q.; Emwas, N.; Al-Kerm, R.; Al-Kerm, R. The inhibitory role of curcumin derivatives on AMPA receptor subunits and their effect on the gating biophysical properties. Eur. J. Pharm. Sci. 2019, 136. [CrossRef]

8. Rogawski, M.A. AMPA receptors as a molecular target in epilepsy therapy. Acta Neurol. Scand. 2013, 127, 9-18. [CrossRef]

9. Schousboe, A. Glutamate neurotoxicity related to energy failure. In Handbook of Neurotoxicity; Springer: New York, NY, USA, 2014; pp. 1299-1310.

10. Lewerenz, J.; Maher, P. Chronic glutamate toxicity in neurodegenerative diseases-what is the evidence? Front. Neurosci. 2015, 9, 469. [CrossRef]

11. Sharma, R.; Rao, R.; Kumar, S.; Mahant, S.; Khatkar, S. Therapeutic potential of Citronella essential oil: A review. Curr. Drug Discov. Technol. 2018, 15. [CrossRef]

12. Williams, T.; Ince, P.G.; Oakley, A.E.; Shaw, P.J. An immunocytochemical study of the distribution of AMPA selective glutamate receptor subunits in the normal human motor system. Neuroscience 1996, 74, 185-198. [CrossRef]

13. Wang, B.; Zhang, G.; Yang, M.; Liu, N.; Li, Y.X.; Ma, H.; Ma, L.; Sun, T.; Tan, H.; Yu, J. Neuroprotective effect of anethole against neuropathic pain induced by chronic constriction injury of the sciatic nerve in mice. Neurochem. Res. 2018, 43, 2404-2422. [CrossRef] [PubMed]

14. Traynelis, S.F.; Wollmuth, L.P.; McBain, C.J.; Menniti, F.S.; Vance, K.M.; Ogden, K.K.; Hansen, K.B.; Yuan, H.; Myers, S.J.; Dingledine, R. Glutamate receptor ion channels: Structure, regulation, and function. Pharmacol. Rev. 2010, 62, 405-496. [CrossRef] [PubMed]

15. Wright, A.L.; Vissel, B. The essential role of AMPA receptor GluR2 subunit RNA editing in the normal and diseased brain. Front. Mol. Neurosci. 2012, 5, 34. [CrossRef]

16. Dong, X.-X.; Wang, Y.; Qin, Z.-H. Molecular mechanisms of excitotoxicity and their relevance to pathogenesis of neurodegenerative diseases. Acta Pharmacol. Sin. 2009, 30, 379-384. [CrossRef]

17. Yennawar, M.; White, R.S.; Jensen, F.E. AMPA receptor dysregulation and therapeutic interventions in a mouse model of CDKL5 Deficiency Disorder. J. Neurosci. 2019, 39, 4814-4828. [CrossRef]

18. Smeriglio, A.; Alloisio, S.; Raimondo, F.M.; Denaro, M.; Xiao, J.; Cornara, L.; Trombetta, D. Essential oil of Citrus lumia Risso: Phytochemical profile, antioxidant properties and activity on the central nervous system. Food Chem. Toxicol. 2018, 119, 407-416. [CrossRef]

19. Throne Jones, T.; Reis, D. Safe Essential oil practice in cancer centers. Oncol. Issues 2018, 33, 48-54. [CrossRef]

20. Sharifi-Rad, M.; Salehi, B.; Sharifi-Rad, J.; Setzer, W.N.; Iriti, M. Pulicaria vulgaris Gaertn. essential oil: An alternative or complementary treatment for Leishmaniasis. Cell. Mol. Biol. 2018, 64, 18-21. [CrossRef]

21. Steinhoff, B.J.; Hübers, E.; Kurth, C.; Jürges Kehl-Kork, U. Plasma concentration and clinical effects of perampanel-The Kork experience. Seizure 2019, 67, 18-22. [CrossRef]

22. Pascuzzi, R.M.; Shefner, J.; Chappell, A.S.; Bjerke, J.S.; Tamura, R.; Chaudhry, V.; Clawson, L.; Haas, L.; Rothstein, J.D. A phase II trial of talampanel in subjects with amyotrophic lateral sclerosis. Amyotroph. Later. Scler. 2010, 266-271. [CrossRef] [PubMed]

23. Winkelman, W.J. Aromatherapy, botanicals, and essential oils in acne. Clin. Dermatol. 2018, 36, $299-305$. [CrossRef] [PubMed]

24. Kennedy, D.; Okello, E.; Chazot, P.; Howes, M.J.; Ohiomokhare, S.; Jackson, P.; Haskell-Ramsay, C.; Khan, J.; Forster, J.; Wightman, E. Volatile Terpenes and brain function: Investigation of the cognitive and mood effects of mentha $\times$ Piperita, L. essential oil with in vitro properties relevant to central nervous system function. Nutrients 2018, 10, 1029. [CrossRef] [PubMed] 
25. Krieger, J.; Lee, J.Y.; Greger, I.H.; Bahar, I. Activation and desensitization of ionotropic glutamate receptors by selectively triggering pre-existing motions. Neurosci. Lett. 2018, 700, 22-29. [CrossRef]

26. Chen, Z.; Wang, X.; Ashraf, U.; Zheng, B.; Ye, J.; Zhou, D.; Zhang, H.; Song, Y.; Chen, H.; Zhao, S.; et al. Activation of neuronal $\mathrm{N}$-methyl-d-aspartate receptor plays a pivotal role in Japanese encephalitis virus-induced neuronal cell damage. J. Neuroinflamm. 2018, 15, 238-244. [CrossRef]

(C) 2019 by the authors. Licensee MDPI, Basel, Switzerland. This article is an open access article distributed under the terms and conditions of the Creative Commons Attribution (CC BY) license (http://creativecommons.org/licenses/by/4.0/). 